\title{
CAMPUSMOVIL.NET. LA PRIMERA RED SOCIAL UNIVERSITARIA VÍA DISPOSITIVOS MÓVILES DE IBEROAMÉRICA. UN ESTUDIO DE CASO
}

\author{
(MOBILE CAMPUS. THE FIRST COLLEGE SOCIAL NETWORKING VIA MOBILE DEVICES IN \\ LATIN AMERICA. A CASE STUDY)
}

\author{
Hugo Pardo Kuklinski \\ Grup de Recerca d'Interaccions Digitals, Universitat de Vic (España)
}

\section{RESUMEN}

El proyecto de la spin-off CampusMovil.net es el resultado de un proceso de investigación + desarrollo de 18 meses de duración que finaliza en el diseño de una red social vía dispositivos móviles para la comunidad universitaria iberoamericana, con acceso exclusivo a través de la cuenta de correo electrónico asignada por la universidad a sus miembros. Se trata de un campus virtual no-oficial, basado en el uso bajo dispositivos móviles.

CampusMovil.net aspira a cubrir necesidades no resueltas por parte de la comunidad universitaria relacionadas con la falta de un servicio ubicuo y con acceso a Internet para múltiples funciones de uso diferencial al que se produce desde los ordenadores personales dentro del campus y en las propias aulas.

En este artículo se explica el desarrollo inicial del proyecto, su alcance y su estrategia de posicionamiento en el mercado de la innovación educativa en la región.

Palabras clave: CampusMovil.net, dispositivos móviles, Mobile Web 2.o, contenidos generados por el usuario, m-learning, innovación docente.

\begin{abstract}
The proposed spin-off of Mobile Campus is the result of an 18 month research and development project to design a social network via mobile devices for the Spanish and Latin American academic
\end{abstract}


communities, with exclusive access via university assigned email accounts. Mobile Campus aims to meet yet the needs of the university community for ubiquitous Internet access to on campus and inclassroom. personal computers. This article discusses the initial development of the project, its scope and the strategy to position it in the market for educational innovation in the region.

Keywords: Campus Móvil, mobile devices, Mobile Web 2.o, user generated contents, mlearning, Bologna Process.

El proyecto CampusMovil.net se ubica en la intersección de tres áreas de conocimiento y de negocio: las aplicaciones Web 2.0, los dispositivos móviles y las políticas de innovación docente del Espacio Europeo de Educación Superior. El Bologna Process ${ }^{1}$ sugiere cambios en la estructura curricular europea, otorgando un énfasis especial a la innovación en los usos de las tecnologías como valor esencial de las nuevas estrategias pedagógicas.

CampusMovil.net es una red social en línea vía dispositivos móviles para la comunidad universitaria iberoamericana, con acceso exclusivo y transparente a través de la cuenta de correo electrónico asignada por la universidad a sus miembros. Su utilización es gratuita por parte de los usuarios registrados y no existe ningún tipo de pago tanto para crear, gestionar o compartir servicios y contenidos. El único coste es el derivado del consumo de Internet móvil en los dispositivos, con pago a las operadoras telefónicas según el contrato de uso que tenga cada usuario.

El proyecto CampusMovil.net nace en Stanford University en 2007 a partir de la investigación "Mobile devices and Web 2.o applications. Towards to design a prototype of university teaching innovation" financiada a través del Ministerio de Educación y Ciencia de España ${ }^{2}$. Por otra parte, la investigación "Comunicación y dispositivos móviles en Cataluña: actores, contenidos y tendencias” (2007-2008) del Grup de Recerca d'Interaccions Digitals (GRID-UVic) y financiada por el Consell d'Audiovisual de Catalunya (CAC) contribuyó a un mejor conocimiento del mercado español y permitió darle forma definitiva a la implementación del producto.

CampusMovil.net es el principal producto de la spin-off Funky Mobile Ideas SL, y cuenta con el soporte inicial del Programa Capital Génesis y del Programa Innova del Centre d'Innovació i Desenvolupament Empresarial (CIDEM), un vivero de empresas dependiente de la Generalitat de Catalunya. El prelanzamiento de la aplicación (en formato beta, bajo invitación individual y cerrada) fue el pasado 3 de noviembre de 2008 y su lanzamiento definitivo en España está previsto para septiembre de 2009. En tanto el lanzamiento en Latinoamérica será gradual por países y esta planificado a partir de 2010. 
Este artículo narra la experiencia de Mobile Web 2.o en el ámbito universitario desde la teoría hasta la implementación y propone recorrer los aspectos conceptuales y de desarrollo del proyecto.

\section{ANTECEDENTES. EL MARCO TEÓRICO}

La mobile Internet es -excepto en escasos países asiáticos y del norte de Europaun mercado en formación y con dificultades, como el alto coste de la conectividad para los usuarios, la lentitud en la navegación web por limitaciones de ancho de banda, la escasa autonomía de las baterías en los dispositivos y la carencia de un hábito de consumo, entre otros factores. Sin embargo las predicciones de los expertos (Castells et al, 2007; Pardo Kuklinski, Brandt y Puerta, 2008; Jaokar y Fish, 2006; Levinson, 2004, Steinbock, 2005, 2003; Wilson, 2006; Silver, 2007; Groebel, Noam, Feldmann, 2006) indican que será uno de los mercados tecnológicos y de consumo de mayor expansión en los próximos años, especialmente de la mano de las aplicaciones colaborativas.

Si bien en todos los mercados mundiales existe un crecimiento sostenido del consumo Web en dispositivos móviles, la especificidad de su uso atiende ciertas características que deben tenerse en cuenta a la hora de promover un nuevo producto como CampusMovil.net. Los usuarios "en movimiento" quieren ser entretenidos con contenidos cortos, directos y amigables. La oferta debe girar alrededor de servicios y contenidos de alto valor añadido en situaciones específicas contextuales relevantes basadas en la ubicuidad, en la captación del punto de inspiración y en los servicios basados en la ubicación; todo sincronizado con aplicaciones web complementarias.

Es imprescindible tener en cuenta la evolución de un "always-on prosumer" con quienes todas las marcas y empresas quieren conectar y seducir. Un tipo de usuario que está impulsando la industria tecnológica, especialmente en el incipiente mercado de la mobile Internet y en el terreno casi virgen de la mobile Web 2.o.

En la línea de lo expresado en los recientes párrafos, dos investigaciones fueron el antecedente más directo de este proyecto. El trabajo Mobile Web 2.o. Theoreticaltechnical framework and developing trends ${ }^{3}$ (Pardo Kuklinski, Brandt, Puerta, 2008) describe la convergencia de aspectos técnicos y de consumo que permiten la hibridación entre dispositivos móviles y Web 2.o, generando un nuevo espacio simbólico denominado mobile Web 2.o. Esta investigación traza un panorama teórico y técnico que permita introducir al lector en el fenómeno de la mobile Web 2.o. Para 
ello, se hace énfasis en los aspectos centrales de la evolución de los teléfonos móviles hacia dispositivos con uso centrado en las aplicaciones colaborativas vía Internet. Además se analizan las dificultades y limitaciones de la industria, los siete principios de la Web 2.0 adaptados a los dispositivos móviles, y los aspectos de productos, contenidos de un mercado incipiente pero en franca evolución.

Cinco son las ideas principales de la investigación:

- Los dispositivos móviles permiten capturar contenidos desde el punto de inspiración y la Web 2.o le agrega el principio de la inteligencia colectiva a través de una taxonomía creada por los usuarios, promoviendo una nueva Mobile Data Industry a tono con la ética de la arquitectura de la participación y más allá del text messaging, ringtones y otros recursos promovidos por las operadoras.

- La limitación de los terminales móviles está más en las limitaciones que imponen los actores del mercado y en sus formas de uso, que en su propia tecnología. La economía de la industria se ha basado en relaciones de poder operador-fabricante por las cuales los administradores de las redes incorporan servicios propietarios del fabricante totalmente dependiente de su red, sin permitir el acceso a terceros desarrolladores.

Esta lógica comercial crea monopolios de facto en los que el usuario subscriptor carece de opciones y ha impedido hasta ahora la conformación de un ecosistema de aplicaciones y servicios que redunden en el incremento de valor de los dispositivos móviles como terminales multimedia. La sociedad operador-fabricante se basa en mecanismos que impiden tanto a los usuarios como a terceros desarrolladores la instalación de software adicional, generalmente mediante la no publicación de APIs, librerías o descripción interna del funcionamiento del sistema operativo.

- Es previsible que la evolución de las tecnologías y del software impulsen la adopción de aplicaciones de navegación web totalmente abiertas para dispositivos móviles. Es de esperar que, como ya sucedió en los sistemas de informática personal, los fabricantes se enfoquen en la creación de extensiones en los navegadores de forma semi-propietarias (API abierto, back-end cerrado o bloqueado por patentes) para la creación cautiva de aplicaciones diferentes en sus modelos de terminal.

- Los contenidos creados en dispositivos móviles e integrados a la Web 2.o podrían modificar el balance de poder en la industria de los medios. El usuario no es 
un número, sino un tag. Las etiquetas pueden proveer un modo de mapear los múltiples números de nuestra vida en forma más natural e intuitiva, liberando al usuario de las restricciones de las operadoras de redes.

La localización es un complejo ejercicio de las redes móviles tradicionales. La mobile Web 2.o está destinada a ser una red mundial móvil vía Web con acceso multilenguaje, sin roaming, ni llamadas internacionales.

- La mobile Web 2.o conducirá los servicios basados en la ubicación, ya que ésta es la cualidad distintiva esencial de los móviles en donde otras herramientas no pueden competir. También plantea la búsqueda móvil en forma diferente a la que se utiliza desde los ordenadores, con énfasis en contexto de tiempo, evento y lugar. Además existe una baja capacidad de serendipity y menor paciencia del usuario.

Por otra parte, el proyecto Comunicación y dispositivos móviles en Cataluña: actores, contenidos y tendencias ${ }^{4}$ (Scolari, Navarro, Pardo Kuklinski, García Medina, Soriano; 2008) fue esencial para comprender la situación del mercado español y adaptar el desarrollo de la aplicación a la realidad y al futuro del ecosistema mobile. La investigación estuvo orientada a explorar la configuración de los dispositivos móviles como un nuevo medio de comunicación en el entorno catalán y a observar cómo se está forjando un sector de la producción de contenidos comunicativos desde el que se alumbran nuevos tipos de contenidos y nuevas gramáticas para su expresión.

Dicho trabajo exploratorio (23 entrevistas a protagonistas del sector) tuvo el objetivo de diseñar un mapa de los actores, contenidos y tendencias del sector de las comunicaciones para teléfonos móviles en Cataluña y, específicamente, en los sectores de contenidos informativos, educativos y de entretenimiento. Además de procurar establecer los puntos cardinales del mapa con definiciones de los conceptos clave, también se ha preocupado por dibujar las tendencias y dinámicas de cambio hacia las que se dirige la industria móvil.

Las cinco ideas principales extraídas de la investigación son:

- La comunicación móvil en Cataluña está viviendo un momento ideal para experimentar nuevas formas de comunicación y modelos de negocios. Se trata de una fase de gran dinamismo y efervescencia a nivel global. Si hasta ahora la producción para el formato móvil se ha centrado en contenidos adaptados de 
otros medios, los entrevistados coinciden en que el futuro será de los contenidos y servicios específicos para dispositivos móviles que aprovechen las características del medio.

- Existe una enorme variedad de dispositivos de comunicación móvil, lo cual dificulta la creación de contenidos y servicios. Los diferentes formatos, dimensiones, sistemas operativos y modelos son un límite a la producción. Aunque la Internet móvil -con navegadores web estandarizados- rompe con esas limitaciones basadas en las características técnicas de los dispositivos y en el actual modelo operador-dependiente.

- Todos los entrevistados son optimistas respecto al futuro de la comunicación móvil y demuestran un entusiasmo similar al que hace una década y media motivaba a los empresarios de la World Wide Web. Otra cosa que comparten los empresarios del mundo móvil con los pioneros de la Web es el sentimiento de estar abriendo camino, de contribuir a la invención de un mercado hasta ahora inexistente.

- Actualmente se vive un momento de plena expansión del mobile marketing, donde se comienza a utilizar como parte de las campañas publicitarias de las empresas, ya que favorece la fidelización del cliente, aumenta la imagen de marca, incentiva la repetición de compra, dirige tráfico al punto de venta y establece un nuevo canal de comunicación entre el usuario y la marca.

- Los entrevistados coinciden en que los SMS seguirán siendo por un tiempo el producto más redituable de la comunicación móvil, pero saben que una nueva generación de contenidos audiovisuales, interactivos y colaborativos está a punto de entrar con fuerza en el mercado español. Mientras se espera este salto, se experimenta con ellos para comprender mejor sus gramáticas y posibilidades de negocios.

\section{EL PRODUCTO}

\section{www.campusmovil.net}

A diferencia de otras aplicaciones que se ofrecen desde la redundancia en la oferta de servicios, CampusMovil.net aspira a cubrir necesidades no resueltas por parte de la comunidad Universitaria Española y Latinoamericana relacionadas con la falta de un servicio ubicuo y con acceso a Internet para múltiples funciones de uso 
diferencial al que se produce desde los ordenadores personales dentro del campus y en las propias aulas. Su novedad es que se trata de un tipo de aplicación pensada para el consumo sobre dispositivos móviles, con complementariedad en usos de escritorio, y no a la inversa, como la mayoría de productos conocidos. Su condición de red cerrada universitaria y con énfasis en la navegación vía dispositivos móviles es su estrategia de diferenciación en el mercado.

La ventaja está en la integración de herramientas existentes en otros mercados relacionados con la telefonía móvil, aunque todos en fase inicial de desarrollo y ninguno dirigido al público universitario, lo cual transforma a CampusMovil.net en un campus virtual no-oficial diseñado para acceder desde el móvil. La idea es desarrollar un tipo de plataforma inexistente en el ecosistema académico español, y que puede funcionar como un excelente complemento de los campus virtuales tradicionales.

CampusMovil.net propone un espacio de consumo y producción entre institución y alumnos tanto a nivel de interacción como a nivel pedagógico y lúdico, a tono con las exigencias de un entorno universitario con crecientes necesidades de conectividad, ubicuidad y adaptación creativa a las nuevas tecnologías. Además, ofrece un servicio básico atractivo donde hoy existe un vacío de mercado y así estar preparados comercialmente y a nivel de implementación para cuando el mercado de consumo de la mobile Internet comience a crecer en España y Latinoamérica y alcance su punto de maduración, previsto para 2010/2011.

\section{Proposición de valor}

- Producto diseñado para el mercado español universitario, con una fase de expansión hacia el entorno iberoamericano prevista para la tercera fase (septiembre 2010).

- El mercado español de consumo de mobile Internet es aún muy débil, especialmente el grupo etario entre 18 y 30 años, el público objetivo de CampusMovil.net. Entre los principales motivos se encuentran las altas y poco transparentes tarifarias de conexión a Internet vía dispositivos móviles, un necesario período de adaptación al uso multimedia de los nuevos dispositivos $3 \mathrm{G}$ y la novedad de este tipo de consumo. Pero -como se señaló en el marco teórico- se espera un sostenido crecimiento de dicho mercado hasta su punto de maduración en 2010. 
CampusMovil.net cubrirá tres necesidades existentes:

- Captar el punto de inspiración en el entorno académico.

- Aprovechar el tiempo sin disponibilidad de computación (transporte público, tiempo entre clase y clase, bibliotecas, espacios públicos fuera del campus) para seguir interactuando con la comunidad universitaria, tanto a nivel de servicios como de gestión del conocimiento con usos académicos.

- Generar retazos de información para ser recuperados y reutilizados en otros entornos.

Además de ser una comunidad universitaria cerrada, el producto está pensado para uso en dispositivos móviles, aunque también tendrá un soporte web tradicional para usos integradores (complementariedad mobile Web / web desktop). Esos dos elementos son su estrategia de diferenciación. Los trámites más complejos como suscripción inicial, usos comunitarios de consumo extenso, recuperación de retazos de información generados desde la aplicación móvil o los canales de comunicación grupo a grupo, estarán reservados al uso vía web desktop.

\section{Conceptos del producto. Servicios}

Concepto 1. Partnership con las universidades

Invitar a las universidades españolas -y posteriormente a las latinoamericanasa sumarse al uso de la aplicación, ofreciéndoles a sus cuerpos de gestión y profesores la implementación gratuita de servicios administrativos y académicos basados en las necesidades de los usuarios enunciadas anteriormente. Se trata de la integración de servicios del campus virtual que permitan su adaptación a entornos de uso de dispositivos móviles.

\section{Propuesta de servicios y contenidos}

Noticias de última hora y tablón de anuncios breves / Ausencia de profesores / Demora en la entrega de trabajos / Info sobre exámenes / Agenda de eventos on-campus y off-campus, basados en la proximidad / Notas / Respuestas breves a solicitudes de alumnos, tipo FAQ's / Servicios de audio, texto y video sobre actividades académicas / Servicios para novatos / Alertas generales / Alertas por problemas de seguridad / Programa Mentor-Erasmus. 
Concepto 2. Exclusividad, identidad transparente y perfiles reales de los usuarios. Acceso gratuito a la red mediante el correo electrónico que la universidad otorga a sus miembros.

\section{Propuesta de servicios y contenidos}

Los miembros de la comunidad CampusMovil.net podrán -ya sea desde su página personal o desde los espacios públicos- consumir, compartir o subir cuatro tipos de archivos: documentos, fotos, audios y video (todos con la data del título, autor, breve descripción y etiquetas).

\section{Concepto 3. Voz igual a valor}

Promover el uso de podcasts y videocasts de corta duración en la relación docente-alumno y alumno-alumno. La tecnología $3 \mathrm{G}$ en los dispositivos móviles no es capaz de trasmitir contenidos multimedia de alta calidad, pero si de promover un servicio de podcast estilo iTunes $U^{5}$. Ayudar a las facultades a crear un espacio similar y luego facilitarles la producción de contenidos mediante una plataforma común y un patrón de desarrollo de fácil uso.

\section{Concepto 4. Textos cortos en movilidad}

Promover la lectura y producción de textos cortos en situaciones de movilidad, ya sea a nivel de apuntes, de agenda o de microblogging tipo Twitter/Jaiku (cada miembro de la comunidad tiene en su página personal un espacio propio para introducir texto de no más de 20 palabras, bajo preguntas como. ¿Qué necesito para la clase de mañana? o ¿¿ué haré esta tarde luego de clase?

Concepto 5. Producción y recuperación de retazos de información (snippets) Producir (en la aplicación mobile Web) y luego recuperar y reutilizar (en el website tradicional) retazos de información, teniendo en cuenta la captación del punto de inspiración y a modo de notas post-it.

\section{Propuesta de servicios y contenidos}

Ideas surgidas en la clase / Data que surge en la interacción en espacios públicos sin acceso a ordenadores / Frases relevantes de los profesores en clase / ayudamemoria en reuniones de investigación / Todo tipo de retazos de información que puedan ser útiles para recuperar posteriormente / 


\section{Su monetización}

La pregunta clave del modelo de negocio de CampusMovil.net es ćcómo monetizar una nueva red social cerrada (limitada al entorno español y a la comunidad universitaria) y centrada en el uso en dispositivos móviles?

Bryant Urstadt, uno de los analistas más críticos con la economía Web 2.o señala (Technology Review, 2008) ${ }^{6}$ que existen variables de burbuja financiera similares a las que precedieron a la gran crisis de Internet a comienzos de 2001. Describe a los emprendimientos como financieramente ineficientes, con una estrategia de volverse grandes con rapidez, construir la base de usuarios y esperar que el dinero llegue, desde una IPO (Initial public offering) ${ }^{7}$, desde anuncios, venta de acciones u otro mecanismos.

Aunque es necesario tener en cuenta este análisis en la construcción de modelos de negocios en el incipiente y atractivo mercado de la mobile Web 2.0, existen variables que no se toman en cuenta para trazar este paralelismo. La principal es que, a diferencia del período 1994-2001- la Web es el medio dominante de socialización para los jóvenes de 15 y 30 años (con porcentajes mucho mayores en jóvenes con formación universitaria), lo cual dibuja un escenario y un tipo de interacción inexistente hace una década, y que facilita y amplifica las posibilidades de aproximación al target universitario por parte de las empresas. Las redes sociales son la actividad de más rápido crecimiento a nivel de usuarios de la Web. Y las redes sociales móviles están cogiendo impulso para fortalecer aún más al mercado de la inteligencia colectiva, incorporándole ubicuidad a la práctica social.

Los nuevos actores aprendemos de los errores pasados. El mayor aprendizaje que los promotores de CampusMovil.net pueden aportar a su Plan de Negocio es la predominancia de tres virtudes imprescindibles para que una red social móvil pueda monetizar el proyecto: targeting y exclusividad a nivel de conformación de la red, flexibilidad técnica y a nivel de interfaz para darle al usuario lo que requiere y costes mínimos para solventar un crecimiento lento y sostenido de la aplicación sin depender de las ayudas externas ni ahogarse financieramente a corto plazo. El targeting y la exclusividad a nivel de diseño del ecosistema permite concentrar la oferta publicitaria y de servicios, personalizando la publicidad con una relación CPM (cost per mille) más productivo para los anunciantes. A diferencia del tipo de consumo de las redes sociales abiertas como Facebook, MySpace, Orkut o Bebo; los usuarios consumen las redes cerradas para obtener y brindar información especifica y existe una menor dispersión de los mensajes. No se trata de buscar amigos, porque los amigos ya están en el entorno. Así la data es más relevante. 
Aunquelos portales colaborativos abiertos se caracterizan por procurar "volumen" de usuarios, uno de sus puntos débiles es la posibilidad de perder foco, disipar el target hasta hacerlo invisible para los anunciantes y, lo que es peor, hacer que los propios usuarios no sientan identificación ni pertenencia. Entre las principales virtudes de las redes sociales off-line. CampusMovil.net es que está diseñado para ser un equilibrado balance entre un sitio controlado (un campus virtual tradicional) y una red social totalmente abierta.

Pero es evidente que no se puede seducir a los anunciantes en un sitio que la gente no visita. Por eso la primera fase es de incubadora y se basa en la flexibilidad a nivel de plataforma y oferta de servicios, otra virtud para adaptarse a las necesidades de los usuarios y de las instituciones universitarias. Por esto la estrategia en los primeros tres años de desarrollo (y más aún del primer año) es ahorrar costes al máximo con un equipo compacto en carácter de outsourcing y compromiso societario que nos dé cierta autonomía financiera para abocarnos a la tarea de ganar masa crítica de usuarios, mejorar al máximo la aplicación y dar entrada al mercado publicitario en sus múltiples formas.

\section{NOTAS}

1 Acerca de The Bologna Process en http://ec.europa.eu/education/policies/educ/ bologna/bologna en.html

2 Proyecto CampusMovil.net. Mobile devices and Web 2.o applications. Towards to design a prototype of university teaching innovation. Beca Programa José Castillejo. Ministerio de Educación y Ciencia. España.

3 Disponible en International Journal of Interactive Mobile Technologies (Octubre 2008) en http://online-journals.org/i-jim/article/view/535/621

4 Disponible en la web del Consell de l'Audiovisual de Catalunya en http://www.cac. cat/pfw files/cma/recerca/estudis recerca/dispositius m bils.pdf

5 http://www.apple.com/education/itunesu/

6 The Next Bubble? The Future of the Web 2.o, por Bryant Urstadt Technology Review. Agosto 2008. https://www.technologyreview.com/Biztech/20916/

7 http://en.wikipedia.org/wiki/Initial public offering 


\section{REFERENCIAS BIBLIOGRÁFICAS}

Pardo Kuklinski, H.; Brandt, J.; Puerta, J. P. (2008). Mobile Web 2.0. Theoreticaltechnical framework and developing trends. International Journal of Interactive Mobile Technologies (iJIM). October. [en línea] Disponible en: http:// online-journals.org/i-jim/issue/view/16 [consulta 2009, 2 de Julio]
Scolari, C.; Navarro, H.; Pardo Kuklinski, H.; García Medina, I.; Soriano, J. (2008). Comunicació i dispositius mòbils a Catalunya: actors, continguts i tendències. Consell de l'Audiovisual de Catalunya. [en línea] Disponible en: http://www.cac.cat/pfw files/cma/ recerca/estudis recerca/dispositius m bils.pdf [consulta 2009, 2 de Julio]

\section{PERFIL ACADÉMICO Y PROFESIONAL DEL AUTOR}

Hugo Pardo Kuklinski. Investigador y desarrollador de aplicaciones web institucionales y mobile Web 2.0 (Proyecto Campus Móvil - 2008). Doctor en Comunicación, Universidad Autónoma de Barcelona. Profesor del Departamento de Comunicación Digital de la Universitat de Vic y miembro del Grupo de Investigación en Interacciones Digitales (GRID). Autor del libro "Planeta Web 2.o. Inteligencia colectiva o medios fast food" (GRID-Uvic, Flacso México. Barcelona / México DF. 2007). Coordinador del proyecto Mobile devices and Web 2.0 applications. Towards to design a prototype of university teaching innovation (Ministerio de Educación y Ciencia. España). Autor de digitalismo.com CEO y Fundador de CampusMovil.net.

E-mail: hugo.pardo@campusmovil.net

DIRECCIÓN DEL AUTOR:

Hugo Pardo Kuklinski / Funky Mobile Ideas SL

Edifici el Sucre. Carrer Historiador Ramon d'Abadal i de Vinyals, 5, 2a planta. 08500. Vic, Barcelona, España.

Fecha de recepción del artículo: 09/03/09

Fecha de aceptación del artículo: 02/07/09 